УДК 517.955.8

DOI $10.46698 / \mathrm{n} 2399-6862-7231-\mathrm{a}$

\title{
СВОЙСТВА ХАРАКТЕРИСТИК КОЛЕБЛЕМОСТИ СЕРГЕЕВА ПЕРИОДИЧЕСКОГО УРАВНЕНИЯ ВТОРОГО ПОРЯДКА
}

\author{
A. X. Сташ ${ }^{1}$ \\ ${ }^{1}$ Кавказский математический центр АГУ, \\ Россия, 385000, Майкоп, ул. Первомайская, 208, \\ E-mail: aidamir.stash@gmail.com
}

\begin{abstract}
Аннотация. В данной работе изучаются свойства характеристик колеблемости Сергеева решений линейных однородных дифференциальных уравнений второго порядка с непрерывными периодическими коэффициентами. Известно, что верхние (слабые и сильные) показатели колеблемости нулей, корней, гиперкорней, строгих и нестрогих смен знаков совпадают с верхними частотами Сергеева нулей, корней и строгих смен знаков. Аналогичное свойство имеет место и для всех перечисленных нижних характеристик колеблемости Сергеева. Однако верхние характеристики решений линейных однородных дифференциальных уравнений второго порядка с ограниченными коэффициентами не всегда совпадают с нижними. В настоящей работе установлено равенство между всеми характеристиками колеблемости Сергеева на множестве решений уравнения Хилла. Более того, найдена эффективная формула, позволяющая их находить и проводить исследование на устойчивость уравнения Хилла. Кроме того, получена формула, связывающая мультипликаторы уравнения Хилла с нецелой частотой Сергеева. Найдены необходимые и достаточные условия устойчивости частоты уравнения Хилла. При доказательстве результатов настоящей работы осуществлялся переход от декартовых координат к полярным, благодаря чему для полярного угла получаем уравнение, которое можно трактовать как уравнение на торе. В качестве вспомогательного результата установлено равенство между числом вращения и частотой уравнения Хилла.
\end{abstract}

Ключевые слова: уравнение Хилла, дифференциальное уравнение на торе, колеблемость, число нулей, показатель колеблемости, число вращения, частота Сергеева, мультипликатор.

Mathematical Subject Classification (2010): 34C10, 34D05, 34D08.

Образец цитирования: Сташ А. Х. Свойства характеристик колеблемости Сергеева периодического уравнения второго порядка // Владикавк. мат. журн.-2021.-Т. 23, вып. 2.-С. 78-86. DOI: 10.46698/n2399-6862-7231-a.

\section{1. Введение}

Настоящая работа посвящена изучению характеристик колеблемости Сергеева линейных однородных уравнений с периодическими коэффициентами. При изучении периодических уравнений удобно перейти от декартовых координат к полярным, причем для полярного угла получается дифференциальное уравнение, не содержащее полярного радиуса и периодическое по обеим переменным. Последнее позволяет трактовать полученное уравнение как уравнение на торе. Известно, что поведение в целом решений дифференциального уравнения на торе полностью характеризуется числом вращения. В настоящей работе установлено совпадение числа вращения со всеми характеристиками колеблемости Сергеева уравнения Хилла и найдена формула, позволяющя их вычислять по коэффициенту уравнения. Кроме того, рассмотрены вопросы устойчивости этих характеристик на множестве уравнений Хилла.

(C) 2021 Сташ А. X. 


\section{2. Определение характеристик колеблемости Сергеева}

Рассмотрим множество $\mathscr{E}^{2}$ линейных однородных уравнений второго порядка

$$
\ddot{y}+a_{1}(t) \dot{y}+a_{2}(t) y=0, \quad t \in \mathbb{R}_{+} \equiv[0 ; \infty),
$$

задаваемых ограниченными непрерывными функциями

$$
a \equiv\left(a_{1}, a_{2}\right): \mathbb{R}_{+} \rightarrow \mathbb{R}^{2},
$$

с которыми в дальнейшем и будем отождествлять сами уравнения. Множество всех ненулевых решений уравнения $a \in \mathscr{E}^{2}$ обозначим через $\mathscr{S}_{*}(a)$. Далее, звездочкой снизу будем помечать любое линейное пространство, в котором выколот нуль.

Зададим в $\mathbb{R}^{2}$ евклидову норму

$$
|m|=\sqrt{m_{1}^{2}+m_{2}^{2}}, \quad m=\left(m_{1}, m_{2}\right) \in \mathbb{R}^{2},
$$

и превратим множество $\mathscr{E}^{2}$ в топологическое пространство с помощью равномерной нормы

$$
\|a\|=\sup _{t \in \mathbb{R}_{+}} \sqrt{a_{1}^{2}(t)+a_{2}^{2}(t)}, \quad a \in \mathscr{E}^{2} .
$$

ОПРЕДЕЛЕНИЕ 1 [1-4]. Скажем, что в точке $t>0$ происходит строгая (нестрогая) смена знака функции $y: \mathbb{R}_{+} \rightarrow \mathbb{R}$, если в любой окрестности этой точки функция $y$ принимает как положительные (неотрицательные), так и отрицательные (неположительные) значения.

Для момента $t>0$ и функции $y: \mathbb{R}_{+} \rightarrow \mathbb{R}$ введем следующие обозначения:

$\nu^{-}(y, t)$ - число точек ее строгой смены знака на промежутке $(0, t]$;

$\nu^{\sim}(y, t)$ - число точек ее нестрогой смены знака на промежутке $(0, t]$;

$\nu^{0}(y, t)$ - число ее нулей на промежутке $(0, t]$;

$\nu^{+}(y, t)$ - число ее корней (т. е. нулей с учетом их кратности) на промежутке $(0, t]$;

$\nu^{*}(y, t)$ - число ее гиперкратных корней на промежутке $(0, t]$ : при его подсчете каждый некратный корень берется ровно один раз, а кратный - бесконечно много раз.

Далее, для вектора $m \in \mathbb{R}_{*}^{2}$ и вектор-функции $\psi y=(y, \dot{y})$ введем обозначение $\nu^{\alpha}(y, m, t) \equiv \nu^{\alpha}(\langle\psi y, m\rangle, t)$, где $\alpha \in\{-, \sim, 0,+, *\},\langle\psi y(\cdot), m\rangle$ - скалярное произведение.

ОПРеДЕЛЕНИЕ $2[1-3,5,6]$. Верхние (нижние) частоты Сергеева знаков, нулей и корней любого решения $y \in \mathscr{S}_{*}(a)$ при $\gamma \in\{-, 0,+\}$ соответственно зададим формулами

$$
\begin{array}{ll}
\hat{\nu}^{\gamma}(y) \equiv \limsup _{t \rightarrow \infty} \frac{\pi}{t} \nu^{\gamma}(y, t) & \left(\check{\nu}^{\gamma}(y) \equiv \liminf _{t \rightarrow \infty} \frac{\pi}{t} \nu^{\gamma}(y, t)\right), \\
\hat{\nu}^{\gamma}(y) \equiv \limsup _{t \rightarrow \infty} \frac{\pi}{t} \nu^{\gamma}(y, t) & \left(\check{\nu}^{\gamma}(y) \equiv \liminf _{t \rightarrow \infty} \frac{\pi}{t} \nu^{\gamma}(y, t)\right) .
\end{array}
$$

В случае совпадения какой-либо верхней частоты Сергеева решения $y$ с одноименной нижней будем называть ее точной и обозначать $\nu^{\gamma}(y)$, а если дополнительно выполняются еще и равенства $\nu^{-}(y)=\nu^{0}(y)=\nu^{+}(y)$, то будем называть такую частоту абсолютной.

ОПРЕДЕЛЕНИЕ 3 [4, 7]. Верхние (нижние) сильный и слабый показатели колеблемости знаков, нулей, корней и гиперкорней любого решения $y \in \mathscr{S}_{*}(a)$ при 
$\alpha \in\{-, \sim, 0,+, *\}$ соответственно зададим формулами

$$
\begin{array}{ll}
\hat{\nu}_{\bullet}^{\alpha}(y) \equiv \inf _{m \in \mathbb{R}_{*}^{n}} \limsup _{t \rightarrow \infty} \frac{\pi}{t} \nu^{\alpha}(y, m, t) & \left(\check{\nu}_{\bullet}^{\alpha}(y) \equiv \inf _{m \in \mathbb{R}_{*}^{n}} \liminf _{t \rightarrow \infty} \frac{\pi}{t} \nu^{\alpha}(x, m, t)\right), \\
\hat{\nu}_{\circ}^{\alpha}(y) \equiv \limsup _{t \rightarrow \infty} \inf _{m \in \mathbb{R}_{*}^{n}} \frac{\pi}{t} \nu^{\alpha}(y, m, t) & \left(\check{\nu}_{\circ}^{\alpha}(y) \equiv \liminf _{t \rightarrow \infty} \inf _{m \in \mathbb{R}_{*}^{n}} \frac{\pi}{t} \nu^{\alpha}(y, m, t)\right) .
\end{array}
$$

При совпадении сильного или слабого верхнего показателя колеблемости решения $y$ с одноименным нижним будем называть их точными и обозначать $\nu_{\bullet}^{\alpha}(y)$ или $\nu_{\circ}^{\alpha}(y)$ соответственно, а при совпадении всех его показателей колеблемости - абсолютными.

\section{3. Формулировка основных результатов}

Будем изучать уравнение Хилла

$$
\ddot{x}+p(t) x=0, \quad t \in \mathbb{R}_{+},
$$

в котором $p: \mathbb{R}_{+} \rightarrow \mathbb{R}_{+}$есть непрерывная $\pi$-периодическая функция. В работах $[2,8,9]$ установлено, что на множестве решений уравнений второго порядка все верхние (как и нижние) характеристики колеблемости Сергеева совпадают между собой и их спектры состоят из одного значения. Причем существует решение некоторого уравнения $a \in \mathscr{E}^{2}$, все верхние характеристики колеблемости которого не совпадают с нижними. Однако, это неравенство не имеет места на множестве решений уравнения Хилла.

Теорема 1. Для любого нетривиального решения $x$ уравнения (1) все частоты Сергеева и показатели колеблемости являются абсолютными и совпадают между собой.

Частоту $\omega(p)$ уравнения Хилла $(0, p) \in \mathscr{E}^{2}$ определим как частоту нулей Сергеева произвольного его ненулевого решения.

Запишем уравнение Хилла в виде системы

$$
\dot{x}=y, \quad \dot{y}=-p(t) x .
$$

Пусть $f(t)$ и $g(t)$ решение уравнения (1), удовлетворяющее начальным условиям

$$
f(0)=1, \quad \dot{f}(0)=0, \quad g(0)=1, \quad \dot{g}(0)=0 .
$$

Тогда

$$
U(t)=\left(\begin{array}{cc}
f(t) & g(t) \\
\dot{f}(t) & \dot{g}(t)
\end{array}\right), \quad U(\pi)=\left(\begin{array}{cc}
f(\pi) & g(\pi) \\
\dot{f}(\pi) & \dot{g}(\pi)
\end{array}\right)
$$

- фундаментальная матрица и матрица монодромии системы (2) соответственно. Напомним, что мультипликаторы уравнения (1) - это собственные значения матрицы $U(\pi)$.

Если $\omega(p) \in \mathbb{Z}$, то о его мультипликаторах почти ничего сказать нельзя, кроме того, что они вещественные, а их знак совпадает со знаком $(-1)^{\omega(p)}$. В противном случае по частоте Сергеева однозначно восстаналиваются мультипликаторы уравнения Хилла, как показывает следующая

Теорема 2. Если частота уравнения Хилла $(0, p) \in \mathscr{E}^{2}$ удовлетворяет условию $\omega(p) \notin \mathbb{Z}$, то его мультипликаторы равны $\mu_{ \pm}=e^{ \pm i \omega \pi}$.

В работах $[2,8,9]$ установлено, что на множестве $\mathscr{E}^{2}$ каждая из характеристик колеблемости Сергеева является непрерывной в смысле равномерной нормы на полупрямой $\mathbb{R}_{+}$. Ниже будет показано, что частота Сергеева уравнения Хилла в некоторых случаях не будет меняться, несмотря на произвольные, но достаточно малые возмущения. 
ОПредЕлЕниЕ 4 [10]. Будем называть частоту $\omega(p)$ уравнения Хилла $(0, p) \in \mathscr{E}^{2}$ устойчивой, если для некоторого $\epsilon>0$ и для любого уравнения Хилла $(0, q) \in \mathscr{E}^{2}$, удовлетворяющего оценке $\sup _{t \in \mathbb{R}_{+}}|q(t)-p(t)|<\epsilon$, имеет место равенство $\omega(q)=\omega(p)$. В противном случае будем называть частоту $\omega(p)$ неустойчивой.

Теорема 3. Если частота уравнения Хилла иррациональна, то она неустойчива.

Теорема 4. Если частота уравнения Хилла рациональна и равна $\frac{s}{k}$, а величина

$$
\int_{0}^{\pi k} \frac{x \ddot{x}(t)-\dot{x}^{2}(t)}{x^{2}(t)+\dot{x}^{2}(t)} d t-\pi s
$$

для любого его решения $x$ имеет один и тот же знак: либо неотрицательный, либо неположительный, - то частота уравнения Хилла неустойчива.

Теорема 5. Для того чтобы частота $\omega(p)$ уравнения Хилла $(0, p) \in \mathscr{E}^{2}$ была устойчива, необходимо и достаточно, чтобы она $\omega(p)=\frac{s}{k}$ была рациональна и для некоторых его решений $x, z$ выполнялось неравенство

$$
\left(\int_{0}^{\pi k} \frac{x \ddot{x}(t)-\dot{x}^{2}(t)}{x^{2}(t)+\dot{x}^{2}(t)} d t-\pi s\right)\left(\int_{0}^{\pi k} \frac{z \ddot{z}(t)-\dot{z}^{2}(t)}{z^{2}(t)+\dot{z}^{2}(t)} d t-\pi s\right)<0 .
$$

При положительном коэффициенте $p$ частота $\omega(p)$ уравнения Хилла $(0, p) \in \mathscr{E}^{2}$ находится без труда, как показывает

Теорема 6. Если $p(t)>0$ на отрезке $[0, \pi]$, то для уравнения Хилла $(0, p) \in \mathscr{E}^{2}$ выполнено равенство

$$
\omega(p)=\frac{1}{\pi} \int_{0}^{\pi} \sqrt{p(\tau)} d \tau
$$

Некоторые результаты настоящей работы были доложены на научном семинаре по качественной теории дифференциальных уравнений в МГУ имени М. В. Ломоносова и анонсированы в [11].

\section{4. Вспомогательные определения и факты}

С любым ненулевым решением $x$ уравнения (1) свяжем непрерывно-дифференцируемую функцию $\varphi: \mathbb{R}_{+} \rightarrow \mathbb{R}$, значение которой в точке $t$ равно некоторому значению угла, образуемого вектором $(x(t), \dot{x}(t))$ с положительным направлением оси абсцисс. Для этого перейдем в системе (2) от декартовых координат $(x, y)$ к полярным координатам $(r, \varphi)$ с помощью равенств $x=r \sin \varphi, y=r \cos \varphi$. При этом для полярного угла получим дифференциальное уравнение

$$
\dot{\varphi}=\cos ^{2} \varphi+p(t) \sin ^{2} \varphi \equiv f(t, \varphi) .
$$

В этом уравнении правая часть периодична по обеим переменным

$$
f(t+\pi, \varphi)=f(t, \varphi), \quad f(t, \varphi+\pi)=f(t, \varphi),
$$

что позволяет рассматривать уравнение (5) как дифференциальное уравнение на торе. Тор $T^{2}$ запишем как произведение двух окружностей $T=S^{1} \times S^{2}$, где $S^{1}$ и $S^{2}$ окружности 
длины $\pi$; с помощью первой определяются параллели, а с помощью второй - меридианы. Согласно теории Пуанкаре - Данжуа дифференциальных уравнений на торе, поведение решений полностью характеризуется числом вращения $\rho$ и некоторым сохраняющим ориентацию гомеоморфным отображением $H$ окружности на себя (см., например [10, c. $147-186])$.

Нетрудно заметить, что функция $f$ ограничена

$$
|f(t, \varphi)| \leqslant \max \{1,|p(t)|\}
$$

и удовлетворяет условию Липшица

$$
|f(t, \varphi)-f(t, \phi)| \leqslant(1+|p(t)|)|\varphi-\phi| .
$$

Оцека (6) гарантирует существование при всех $t$ решения уравнения (5), удовлетворяющего любому начальному условию, а условие Липшица (7) обеспечивает единственность этого решения и его непрерывную зависимость от начальных условий.

Обозначим через $\varphi\left(t, \varphi_{0}\right)$ решение уравнения $(5)$, удовлетворяющее начальному условию $\varphi(0)=\varphi_{0}$. В силу периодичности функции $f$ найдется такое целое $k$, что выполняется

$$
\varphi\left(t, \varphi_{0}+k \pi\right)=\varphi\left(t, \varphi_{0}\right)+k \pi .
$$

Поэтому большая часть классической теории Пуанкаре - Данжуа дифференциальных уравнений на торе переносится и на рассматриваемый нами случай.

ОПредЕлЕниЕ 5 [10]. Число вращения $\rho$ дифференциального уравнения (1) (или (5)) определяется следующим образом:

$$
\rho=\lim _{t \rightarrow+\infty} \frac{\varphi\left(t, \varphi_{0}\right)}{t}
$$

причем написанный предел существует равномерно по $\varphi_{0}$.

ЗАмЕчАниЕ. Число вращения $\rho$ уравнения Хилла (1) неотрицательно.

В самом деле. Так как $f(t, 0) \geqslant 0$ при всех $t$, то $\varphi\left(t, \varphi_{0}\right) \geqslant 0$ при $\varphi_{0} \geqslant 0$ и $0 \leqslant t<+\infty$. Поэтому согласно (8) выполнено неравенство $\rho \geqslant 0$.

Лемма 1 (Основная). Частота $\omega$ уравнения Хилла (1) совпадает с числом его вращения $\rho$.

$\triangleleft$ Так как $p(t) \geqslant 0$ при $0 \leqslant t<+\infty$, то при любом $\varphi_{0} \geqslant 0$ функция $\varphi\left(t, \varphi_{0}\right)$ возрастает.

При обращении $x(t)$ в нуль $\varphi\left(t, \varphi_{0}\right)$ проходит некоторое значение $\pi n$, поэтому $\nu(x, t) \sim$ $\left[\frac{\varphi\left(t, \varphi_{0}\right)}{\pi}\right]$ при $t \rightarrow+\infty$, где $[a]-$ целая часть числа $a$.

Следовательно, для выбранного решения $x$ будем иметь

$$
\omega=\nu(x)=\lim _{t \rightarrow+\infty} \frac{\pi}{t} \nu(x, t)=\lim _{t \rightarrow+\infty} \frac{\pi}{t}\left[\frac{\varphi\left(t, \varphi_{0}\right)}{\pi}\right]=\lim _{t \rightarrow+\infty} \frac{\varphi\left(t, \varphi_{0}\right)}{t}=\rho . \triangleright
$$

Лемма 2. Если частота Сергеева уравнения (1) рациональна $\omega=\frac{s}{k}(k, s \in \mathbb{N})$, то для некоторого его решения $x$ справедливо равенство

$$
\int_{0}^{\pi k} \frac{x \ddot{x}(t)-\dot{x}^{2}(t)}{x^{2}(t)+\dot{x}^{2}(t)} d t=\pi s .
$$


$\triangleleft$ Ориентированный угол между векторами $(x(0), \dot{x}(0))$ и $(x(\pi k), \dot{x}(\pi k))$ равен (см. [12])

$$
\int_{0}^{\pi k} \frac{x \ddot{x}(t)-\dot{x}^{2}(t)}{x^{2}(t)+\dot{x}^{2}(t)} d t
$$

Поэтому равенство $(9)$ на плоскости $(t, \varphi)$ принимает вид

$$
\varphi\left(\pi k, \varphi_{0}\right)-\varphi_{0}=\pi s, \quad \varphi_{0} \in[0, \pi) .
$$

Справедливость этого равенства следует из того, что число вращения уравнения (5) рационально $\rho=\frac{s}{k}$ и теоремы 10.2 из работы [10, с. 154]. $\triangleright$

\section{5. Доказательства основных результатов}

$\triangleleft$ ДоказАТЕЛЬСтво теоремы 1. Вытекает из леммы 1 настоящей работы и $[9$, теорема 4]. $\triangleright$

$\triangleleft$ ДоКАЗАТЕЛЬСТво теОРемы 2. Вытекает из леммы 1 настоящей работы и [13, теорема 3]. $\triangleright$

$\triangleleft$ ДОКАЗАТЕЛЬСТВО ТЕОРЕМЫ 3. Следует из леммы 1 настоящей работы и $[10$, лемма 11.1]. $\triangleright$

$\triangleleft$ ДокаЗАТЕЛЬСтво теОРемы 4. Неравенство (3) на плоскости $(t, \varphi)$ запишется в виде

$$
\Delta\left(\varphi_{0}\right) \leqslant 0 \quad\left(\text { или } \Delta\left(\varphi_{0}\right) \geqslant 0\right) \quad\left(\forall \varphi_{0} \in[0, \pi)\right),
$$

где $\Delta\left(\varphi_{0}\right)=\varphi\left(\pi k, \varphi_{0}\right)-\varphi_{0}-\pi s$.

Следовательно, в силу леммы 1 настоящей работы и [10, с. 178, лемма 11.2], приходим к заключению теоремы 4.

$\triangleleft$ ДокАЗАТЕЛЬСтво теОРемы 5. Неравенство (4) означает, что функция $\Delta\left(\varphi_{0}\right)$ принимает значения разных знаков на полуинтервале $[0, \pi)$. Далее, для завершения доказательства применяем лемму 1 настоящей работы и [10, с. 179 , теорема 11.2]. $\triangleright$

$\triangleleft$ ДоказАтеЛЬСтво теоремы 6. Фиксируем уравнение Хилла $(0, p) \in \mathscr{E}^{2}$ и некоторое его нетривиальное решение $u$. Заметим, что из периодичности и положительности функции $p$ следуют

$$
\int_{0}^{+\infty} \sqrt{p(t)} d t=+\infty, \quad \frac{p(t+c / \sqrt{p(t)})}{p(t)} \rightarrow 1
$$

при $t \rightarrow+\infty$ равномерно на каждом фиксированном ограниченном $c$-интервале прямой $-\infty<c<+\infty$.

Следовательно, из $[14$, с. 411] вытекает эквивалентность функций $\pi \nu(u, t)$ и $\int_{0}^{t} \sqrt{p(\tau)} d \tau$ при $t \rightarrow+\infty$.

Таким образом, получим

$$
\omega(p)=\lim _{t \rightarrow+\infty} \frac{\pi}{t} \nu(u, t)=\lim _{t \rightarrow+\infty} \frac{1}{t} \int_{0}^{t} \sqrt{p(\tau)} d \tau=\lim _{k \rightarrow+\infty} \frac{1}{\pi k} \int_{0}^{\pi k} \sqrt{p(\tau)} d \tau=\frac{1}{\pi} \int_{0}^{\pi} \sqrt{p(\tau)} d \tau . \triangleright
$$

\section{6. Заключение}

В работе проведено исследование показателей колеблемости и частот Сергеева линейного однородного дифференциального уравнения с непрерывными периодическими 
коэффициентами. Доказана идентичность этих характеристик колеблемости на множестве решений уравнений Хилла, установлена взаимосвязь нецелой частоты уравнения Хилла с мультипликаторами. Найдены условия, при которых частота уравнения Хилла не меняется при достаточно малых возмущениях.

А. А. Жукова установила, что число вращения полностью характеризует устойчивость уравнения Хилла. А именно, уравнение Хилла (с различными мультипликаторами) сильно устойчиво (сильно неустойчиво) тогда и только тогда, когда число вращения есть нецелое (целое) неотрицательное число. Основным недостатком этого результата является отсутствие алгоритма нахождения числа вращения. Теория характеристик колеблемости Сергеева бурно развивается с 2004 г., но тем не менее до сих пор не было применений этим характеристикам. Равенство, установленное между числом вращения и характеристиками колеблемости Сергеева уравнения Хилла, а также формула, позволяющая их вычислить (теорема 6), дает возможность применения на практике этих характеристик при исследовании на устойчивость уравнения Хилла.

Благодарность. Автор выражает глубокую благодарность профессору И. Н. Сергееву за постановку задачи и внимание к работе.

\section{Литература}

1. Сергеев И. Н. Определение характеристических частот линейного уравнения // Диф. уравнения.-2004.-Т. 40.-№ 11.-С. 1573.

2. Сергеев И. Н. Определение и свойства характеристических частот линейного уравнения // Тр. сем. им. И. Г. Петровского.-М.: Изд-во Моск. ун-та, 2006.-Вып. 25.-С. 249-294.

3. Сергеев И. Н. Свойства характеристических частот линейных уравнений произвольного порядка // Тр. сем. им. И. Г. Петровского.-М.: Изд-во Моск. ун-та, 2013.-Вып. 29.-С. 414-442.

4. Сергеев И. Н. Замечательное совпадение характеристик колеблемости и блуждаемости решений дифференциальных систем // Мат. сб.-2013.-Т. 204, № 1.-С. 119-138. DOI: 10.4213/sm7928.

5. Барабанов E. А., Войделевич А. С. К теории частот Сергеева нулей, знаков и корней решений линейных дифференциальных уравнений. I // Диф. уравнения.-2016.-Т. 52, № 10.-С. 1302-1320. DOI: $10.1134 /$ S0374064116100034.

6. Быков В. В. О бэровской классификации частот Сергеева нулей и корней решений линейных дифференциальных уравнений // Диф. уравнения.-2016.-Т. 52, № 4.-С. 419-425. DOI: 10.1134/S0374064116040026.

7. Сергеев И. Н. Определение полных частот решений линейного уравнения // Диф. уравнения.2008.- Т. 44, № 11.-С. 1577.

8. Сергеев И. Н. Колеблемость и блуждаемость решений дифференциального уравнения второго порядка // Вестн. Моск. ун-та. Сер. 1. Матем. Механика.-2011.-№ 6.-С. 21-26.

9. Сергеев И. Н. Характеристики колеблемости и блуждаемости решений линейной дифференциальной системы // Изв. РАН. Сер. мат.-2012.-Т. 76, № 1.-P. 149-172. DOI: 10.4213/im5035.

10. Плисс В. А. Нелокальные проблемы теории колебаний.-Москва: Наука, 1964.-367 с.

11. Сташ A. Х. Свойства показателей колеблемости и частот Сергеева уравнения Хилла // Диф. уравнения.-2020.-Т. 56, № 6.-С. 837-838.

12. Сергеев И. Н. Определение характеристик вращаемости решений дифференциальных систем и уравнений // Диф. уравнения.-2013.-Т. 49, № 11.-С. 1501-1503.

13. Жукова A. А. Число вращения как полная характеристика устойчивости уравнения Хилла // Вестн. СамГУ. Естественнонаучн. сер.-2009.-№ 2 (68).-С. 26-32.

14. Хартман Ф. Обыкновенные дифференциальные уравнения.-М.: Мир, 1970.

Статъя поступила 28 июля 2020 г.

СТАШ АЙДАМИР ХАЗРЕТОВИЧ

Кавказский математический центр АГУ,

доцент кафедры математического анализа

и методики преподавания математики

РОССИЯ, 385000, Майкоп, ул. Первомайская, 208

E-mail: aidamir.stash@gmail.com 


\title{
PROPERTIES OF SERGEEV OSCILATION CHARACTERISTICS OF PERIODIC SECOND-ORDER EQUATION
}

\author{
Stash, A. Kh. ${ }^{1}$ \\ ${ }^{1}$ Caucasus Mathematical Center, Adyghe State University, \\ 208 Pervomayskaya St., Maikop 385000, Russia \\ E-mail: aidamir.stash@gmail.com
}

\begin{abstract}
In this paper, we study the properties of the Sergeev oscillation characteristics of solutions of linear homogeneous second-order differential equations with continuous periodic coefficients. It is known that the upper (weak and strong) oscillation of zeros, roots, hyperroots, strict and non-strict sign changes coincide with the upper Sergeyev frequencies of zeros, roots, and strict sign changes. A similar property holds for all of the listed lower characteristics of Sergeev's oscillation. However, the upper characteristics of solutions of linear homogeneous second-order differential equations with bounded coefficients do not always coincide with the lower ones. In the present paper, equality is established between all characteristics of Sergeev's oscillation on the set of solutions of the Hill equation. Moreover, we have found an effective formula that allows us to find them and conduct studies on the stability of the Hill equation. Besides, a formula connecting Hill equation multipliers with non-integer Sergeyev's frequencies is obtained. Necessary and sufficient conditions of the stability of frequency of the Hill's equation are derived. In proving the results, the transition from Cartesian coordinates to polar coordinates was carried out, so that for the polar angle we obtain an equation that can be interpreted as an equation on the torus. As an auxiliary result, equality was established between the rotation number and the frequency of the Hill equation.
\end{abstract}

Key words: Hill's equation, differential equation on a torus, oscillation, number of zeros, exponents of oscillation, rotation number, Sergeev frequency, multiplier.

Mathematical Subject Classification (2010): 34C10, 34D05, 34D08.

For citation: Stash, A. Kh. Properties of Sergeev Oscilation Characteristics of Periodic Second-Order Equation, Vladikavkaz Math. J., 2021, vol. 23, no. 2, pp. 78-86 (in Russian).

DOI: $10.46698 / \mathrm{n} 2399-6862-7231-\mathrm{a}$.

\section{References}

1. Sergeev, I. N. Definition of Characteristic Frequencies of Linear Equation, Differentsial'nye uravneniya [Differential Equations], 2004, vol. 40, no. 11, pp. 1573 (in Russian).

2. Sergeev, I. N. Definition and Properties of Characteristic Frequencies of a Linear Equation, Journal of Mathematical Sciences, 2006, vol. 135, no. 1, pp. 2764-2793. DOI: 10.1007/s10958-006-0142-6.

3. Sergeev, I. N. Properties of Characteristic Frequencies of Linear Equations of Arbitrary Order, Journal of Mathematical Sciences, 2014, vol. 197, no. 3, pp. 410-426. DOI: 10.1007/s10958-014-1723-4.

4. Sergeev, I. N. The Remarkable Agreement Between the Oscillation and Wandering Characteristics of Solutions of Differential Systems, Sbornik: Mathematics, 2013, vol. 204, no. 1, pp. 114-132. DOI: 10.1070/SM2013v204n01ABEH004293.

5. Barabanov, E. A. and Voidelevich, A. S. Remark on the Theory of Sergeev Frequencies of Zeros, Signs and Roots for Solution of Linear Differential Equation. I, Differential Equation, 2016, vol. 52, no. 10, pp. 1249-1267. DOI: 10.1134/S0012266116100013.

6. Bykov, V. V. On the Baire Classification of Sergeev Frequencies of Zeros and Roots of Solutions of Linear Differential Equations Differential Equations, Differential Equation, 2016, vol. 52, no. 4, pp. 413-420. DOI: $10.1134 /$ S0012266116040029.

7. Sergeev, I. N. Definition of Full Frequencies of Solutions of the Linear Equation, Differentsial'nye uravneniya [Differential Equation], 2008, vol. 44, no. 11, pp. 1577 (in Russian).

8. Sergeev, I. N. Oscillation and Wandering of Solution to a Second Order Differential Equation, Moscow University Mathematics Bulletin, 2011, vol. 66, no. 6, pp. 250-254. DOI: 10.3103/S0027132211060052. 
9. Sergeev, I. N. Oscillation and Wandering Characteristics of Solutions of a Linear Differential Systems, Izvestiya: Mathematics, 2012, vol. 76, no. 1, pp. 139-162. DOI: 10.1070/IM2012v076n01ABEH002578.

10. Pliss, V. A. Nelokal'nye problemi teorii kolebanii [Nonlocal Problems of Oscillation Theory], Moscow, Nauka, 1964 (in Russian).

11. Stash, A. Kh. Properties of Exponents of Oscillation and of the Sergeev's Frequencies of the Hill's Equation, Differentsial'nye uravneniya [Differential Equation], 2020, vol. 56, no. 6, pp. 837-838 (in Russian).

12. Sergeev, I. N. Definition of Rotational Characteristic of Solutions of Differential Systems and Equation, Differentsial'nye uravneniya [Differential Equation], 2013, vol. 49. no. 11, pp. 1501-1503 (in Russian).

13. Jukova, A. A. Rotation Number Like Total Characteristic of Stability of Hill's Equation, Vestnik Samarskogo Gosudarstvennogo Universiteta. Estestvenno-Nauchnaya Seriya, 2009, no. 2 (68), pp. 26 -32 (in Russian).

14. Hartman, Ph. Ordinary Differential Equations, 2nd. ed., SIAM Classics in Applied Mathematics, vol. 38, Society for Industrial and Applied Mathematics, Philadelphia, 2002; reprinted from 2nd. ed. Birkhauser 1982; reprinted from original John Wiley \& Sons, 1964.

Received July 28, 2020

Aydamir Kh. Stash,

Caucasus Mathematical Center, Adyghe State University,

208 Pervomayskaya St., Maikop 385000, Russia,

Associate Professor

E-mail: aidamir.stash@gmail.com 